\title{
Mood disorder as a specific complication of stroke
}

\author{
MARSHALF. FOLSTEIN, R I CHARD MA IBERGER, \\ A N D P A U L R. M C H U G H
}

From the Department of Psychiatry and Behavioral Sciences, The Johns Hopkins School of Medicine, Baltimore, Maryland, USA

SUMMARY In an effort to discern whether cerebral vascular injuries provoke specific emotional disturbances, 20 consecutively admitted stroke patients were compared with 10 orthopaedic patients. Both groups were examined for functional disabilities (Activities of Daily Living) and for psychiatric symptoms. Reliable and valid instruments, the Hamilton Rating Scale, the Visual Analogue Mood Scale, the Present State Exam, and the Mini-Mental State Exam were employed to display the psychopathology. More of stroke patients than orthopaedic patients were depressed ( $45 \%$ versus $10 \%$ ) even though the level of functional disability in both groups was the same. Patients with right hemisphere stroke seemed particularly vulnerable and displayed a syndrome of irritability, loss of interest, and difficulty in concentration, in addition to depression of mood $(70 \%$ of right hemisphere stroke patients versus $0 \%$ left hemisphere stroke patients and $0 \%$ orthopaedic patients). We conclude that mood disorder is a more specific complication of stroke than simply a response to the motor disability. We suggest that a controlled trial of antidepressant medication is indicated for patients with this complication.

Persistent and refractory depression of mood has often been reported to follow stroke (Bleuler, 1924; Post, 1961; Adams, 1963; Bell, 1966). This symptom is commonly explained as an expected psychological reaction of the patient to his disability or restrictions in activity. We began to doubt the adequacy of this explanation when we admitted to the Psychiatry Service for the elderly at the Westchester Division of the New York Hospital, a 72 year old physician who, after a stroke manifest by a left hemiparesis, had developed incapacitation, symptoms of irritability and depression persisting for two years. After treatment with antidepressant medication these distressing symptoms were relieved and his interest and enjoyment of life returned. From this experience we were prompted to study the emotional symptoms of a group of patients with stroke, comparing them with the emotional symptoms of patients with non-neurological but disabling conditions. Our purpose was to discover any specific emotional changes after stroke. Such a

Address for reprint requests: Dr Marshal F. Folstein, Director, General Hospital Psychiatry Division, Osler 320, The Johns Hopkins Hospital, 601 N. Broadway, Baltimore, Maryland 21205, USA.

Accepted 29 March 1977 discovery might encourage an aggressive effort at pharmacological treatment of the symptoms perhaps overlooked because of the belief that they are the inevitable consequences of an unhappy circumstance.

\section{Methods}

We studied the consecutive series of admissions to the Burke Rehabilitation Hospital of patients who were disabled by stroke or other conditions but were free of complicating systemic disease and who were suitable for rehabilitation. We excluded patients who had a history of previous neurological disorder and any patients suffering from current medical disorder such as severe congestive heart failure, infection, or poorly controlled metabolic disease, and any patients with stroke producing cranial nerve or cerebellar disorder so as to avoid the inclusion of brain stem infarctions with their potential for disruption of vital cardiorespiratory function. With these exclusions a series of 20 patients with cerebral vascular infarctions (10 right hemisphere and 10 left hemisphere), admitted 30 days after onset of stroke, were selected as were orthopaedically dis- 
abled patients admitted at the same time because of hip fracture or severe arthritis. We can thus report on an experience with 30 patients seen consecutively in a rehabilitation hospital. One of us (RM) examined all patients with standard medical, neurological, and psychiatric methods. All the stroke patients had been diagnosed previously as suffering from a vascular accident in a cerebral hemisphere, and focal disorder of the hemisphere had been discovered previously by such tests as electroencephalograms and brain. scans. Our examinations agreed with these findings in every patient.

Simple scales measuring neurological impairment and performance of activities of daily living were constructed. For the documentation of psychiatric symptoms we carried out a thorough, structured psychiatric interview of proven reliability on all patients, the eighth edition of the present state examination (PSE) as described by Wing et al. (1974). We had become practised in the use of this instrument and reported our capacity with it to demonstrate psychiatric symptoms (Luria and McHugh, 1974). The PSE was scored by the construction of scales and categories thought to reflect differences between groups studied. The 'total PSE score' was obtained by adding scores on all items. Table 1 lists the criteria for the depression and irritability categories.

Table 1 Criteria for depression and irritability categories

Criteria for depression category (8 of following PSE items)

Criteria for irritability category (7 of following PSE items)

Depression or low spirits Crying or wanting to cry

Diurnal variation

Anorexia

Insomnia

Constipation

Slower muddled thinking

Lack of energy

Lack of self-confidence

Non-specific aches and pains

Crying during the interview

Looks and sounds depressed

Noted depressed by the

examining psychiatrist
(Folstein et al., 1975) and the Peabody Vocabulary and Ravens Matrices.

\section{Results}

A greater percentage of stroke patients than orthopaedic patients were in the depression category (Table 2). A trend was noted for the stroke patients to appear more depressed than the orthopaedic patients on the Hamilton Rating Scale and the visual analogue scale (Table 2). A higher incidence of depression was found in the stroke patients statistically matched for physical disability (Activities of Daily Living scores) (Table 3). Although depression was frequent after both right and left hemisphere infarction in the group of stroke patients, the right hemisphere group suffered from an increase in overall psychological symptoms as seen in the increased total PSE scores (Table 2). The increase of symptoms in the right hemisphere patients was characterised particularly by scores on the irritability category. Seven of 10 right hemisphere damaged patients were in the irritability category as compared to none of 10 in the left hemisphere damaged patients (Table 2).

\section{Discussion}

With these methods we discovered that mood disorder is a common and specific symptom of patients recovering from stroke. Since none of the study patients were being evaluated or treated psychiatrically, even though psychiatric consultants were available, we must assume that this disorder had been unrecognised as a psychiatric complication. The plausible hypothesis that may have interfered with recognition and attempt at treatment was that depression in stroke patients is explained adequately by an understandable psychological reaction to motor disability. But this hypothesis is refuted since patients suffering comparable disability from skeletal pathology did not suffer comparable psychiatric symptoms. However, another hypothesis is that infarction of the brain can produce the appearance of a mood disorder. In fact, the appearance of certain affective symptoms after right rather than left hemisphere lesions adds support to such a hypothesis. The mechanism for the appearance of depression after stroke has been suggested by the work of Robinson et al. (1975) who reported that brain stem catecholamines are depleted after experimentally induced cortical cerebral infarction in the rodent and that the behavioural dis-
The visual analogue mood scale, another instruour hands (Folstein and Luria, 1973) and the Hamilton Rating Scale for Depression were employed as well. For measurement of cognitive function we employed our Mini-Mental State 
Table 2 Results of examinations for functional and psychiatric disabilities in stroke and orthopaedic patients

\begin{tabular}{|c|c|c|c|c|}
\hline & All stroke & Left hemisphere & Right hemisphere & Orthopaedic \\
\hline Number of patients & 20 & 10 & 10 & 10 \\
\hline Age (yr) & 63.3 & 60.9 & 64.6 & 67.5 \\
\hline $\operatorname{Sex}(\mathbf{M} / \mathbf{F})$ & $9 / 11$ & $3 / 7$ & $6 / 4$ & $3 / 7$ \\
\hline Depression category (\%) & 45 & 50 & 40 & 10 \\
\hline Hamilton Rating Scale & 12 & 11 & 15 & 8 \\
\hline Visual Analogue Mood Scale & 41 & 42 & 40 & 56 \\
\hline Total PSE score & $45 \pm 6$ & $35 \pm 9$ & $54 \pm 9$ & $20 \pm 3$ \\
\hline Irritability category $(\%)$ & 35 & 0 & 70 & 0 \\
\hline Mini-Mental State & 22 & 21 & 24 & 26 \\
\hline Ravens Matrices & 7 & 7 & 8 & 9 \\
\hline Peabody Vocabulary & 110 & 105 & 118 & 108 \\
\hline Activities of daily living & 42 & 40 & 44 & 57 \\
\hline Neurological impairment & 5 & 6 & 4 & - \\
\hline Duration of illness (days) & 47 & 57 & 39 & 360 \\
\hline
\end{tabular}

Table 3 Analysis of stroke and orthopaedic patients in depression and irritability categories

\begin{tabular}{lcc}
\hline & Stroke & Orthopaedic \\
\hline Number of patients & 8 & 8 \\
Age (yr) & 65 & 70 \\
Sex (M/F) & $4 / 4$ & $2 / 6$ \\
Depressive category (\%) & 50 & 13 \\
Hamilton Rating Scale & 12 & 8 \\
Visual Analogue Mood Scale & 53 & 49 \\
Total PSE score & $44 \pm 7$ & $20 \pm 3$ \\
Irritability category (\%) & 50 & 0 \\
Mini-Mental State & 24 & 26 \\
Ravens Matrices & 8 & 9 \\
Peabody Vocabulary & 117 & 126 \\
Activities of daily living & 56 & 54 \\
\hline
\end{tabular}

order produced by the lesion was altered by desipramine. In view of the possibility suggested by these results a clinical trial of tricyclic antidepressants is indicated. This may document further a specific mood disorder in stroke patients as well as provide knowledge for the relief of these symptoms.

\section{References}

Adams, G. F. (1963). Mental barriers to recovery from strokes. Lancet, 2, 533-537.
Bell, D. S. (1966). Psychiatric aspects of cerebral vascular disease. Medical Journal of Australia, 2, 829-833.

Bleuler, E. (1924). Textbook of Psychiatry, p. 280. Macmillan: New York.

Folstein, M. F., Folstein, S. E., and McHugh, P. R. (1975). 'Mini-Mental State'. Journal of Psychiatric Research, 12, 189-198.

Folstein, M. F., and Luria, R. E. (1973). Reliability, validity and clinical application of the visual analogue mood scale. Psychological Medicine, 3, 479-486.

Luria, R., and McHugh, P. R. (1974). The reliability and clinical utility of the present state examination. Archives of General Psychiatry, 30, 866-871.

Post, F. (1961). The significance of affective symptoms in old age: a follow-up of 100 patients. MD Thesis, University of London.

Robinson, R. G., Shoemaker, W. J., Schlumpf, M., Valk, T., and Bloom, F. E. (1975). Effect of experimental cerebral infarction in rat brain on catecholamines and behaviour. Nature, 255, 332334.

Wing, J. K., Cooper, J. E., and Sartorius, N. (1974). The Measurement and Classification of Psychiatric Symptomatology. Cambridge University Press: Cambridge. 